\title{
An Optimization Method for Semilinear Parabolic Relaxed Constrained Optimal Control Problems
}

\author{
Basil Kokkinis ${ }^{1}$ \\ ${ }^{1}$ Department of Mathematics, School of Applied Mathematical and Physical Sciences, National Technical University, Athens, Greece
}

\author{
Article Info \\ Keywords: Conditional descent \\ method, Discretization, Optimal \\ control, Relaxed controls, Semilinear \\ parabolic equations, State constraints \\ 2010 AMS: 49M05, 49M25, 65N30 \\ Received: 11 November 2019 \\ Accepted: 7 April 2020 \\ Available online: 30 June 2020
}

\begin{abstract}
This paper addresses optimal control problems governed by semilinear parabolic partial differential equations, subject to control constraints and state constraints of integral type. Since such problems may not have classical solutions, a relaxed optimal control problem is considered. The relaxed control problem is discretized by using a finite element method and the behavior in the limit of discrete optimality, admissibility and extremality properties is studied. A conditional descent method with penalties applied to the discrete problems is proposed. It is shown that the accumulation points of sequences produced by this method are admissible and extremal for the discrete problem. Finally, numerical examples are given.
\end{abstract}

\section{Introduction}

In the absence of any convexity assumptions, optimal control problems, in general, have no classical solutions. To study them, they usually need to be reformulated to their corresponding relaxed form. Warga [1], Roubíček [2] and Fattorini [3] have extensively studied the concept of relaxation on optimal control problems. Relaxation had been introduced initially to prove the existence of optimal controls and then to derive necessary optimality conditions. Additionally, relaxed controls are used as a tool to develop optimization methods (Warga [4], Chryssoverghi et al. [5]) and discrete approximation schemes (Chryssoverghi et al. [6], Roubíček [7], Azhmyakov et al. [8]). Relaxed controls have been applied to optimal control problems for systems defined by PDEs in [3], [2] as well as in many papers, among them [6], [9]-[13]. In particular, Arada and Raymond in [9] prove existence and a Pontryagin's minimum principle for relaxed solutions of state-constrained relaxed optimal control problems governed by semilinear elliptic equations under a stability condition. The approximation of similar problems was studied by the same authors in [10] and by Casas in [11]. Chryssoverghi and Bacopoulos in [6] present approximation results for relaxed semilinear parabolic optimal control problems. In [12] relaxed controls have been used to develop iterative optimization methods applied directly on a relaxed problem. Finally, Luan in [13], using relaxed controls obtains some results on the nonexistence and existence of multisolution semilinear elliptic optimal control problems.

In this paper, an optimal control problem with distributed control is considered for systems defined by a semilinear parabolic PDE, in the presence of constraints on the control and the state. The parabolic equation has two separate semilinear terms in order to allow more general assumptions, monotonicity for the term on the left-hand side and Lipschitz continuity for the term on the right-hand side. The state constraints depend both on the state and its gradient and are of integral type. The cost functional depends also on the state gradient. Convexity assumptions are not imposed, so this problem may have no classical solutions. To deal with this, the problem is reformulated in its relaxed form using relaxed controls. The state equation in relaxed 
form is then discretized in space using a Galerkin finite element method (semi-discretization). The spatial discretization is done with continuous piecewise linear functions. The controls are approximated by piecewise constant relaxed ones. Necessary conditions for optimality are stated for the discrete relaxed problem. Then it is shown that sequences of optimal (resp. extremal) relaxed controls for the discrete problem have subsequences which converge to optimal (resp. extremal) controls for the continuous relaxed problem. Next, an algorithm based on a penalized conditional descent method is proposed, applied to the discrete problems, which generates Gamkrelidze controls. It is shown that accumulation points of sequences constructed by the algorithm satisfy the necessary conditions for optimality for the discrete problem and such accumulation points always exist. For implementation reasons relaxed controls have to be approximated by classical ones. So, using standard techniques, the Gamkrelidze controls computed by the above method can be approximated by piecewise constant classical ones, see [5]. Thus the above method using relaxed controls has all the theoretical advantages of them and gives us at last, through the above-mentioned approximation, classical controls. Finally, two numerical examples are presented.

The novelty points of this paper are: (i) the study of such nonconvex optimal control problems with relaxation, (ii) the discretization of such problems, and (iii) the construction of methods applied to the discrete problem with relaxed controls. In order to solve these problems numerically one must necessarily disretize them and then apply some optimization method to the resulting discrete problem. Since the structures of the continuous and the discrete problems are basically different it is necessary to know if discrete optimality (or extremality) carries over in the limit to continuous optimality (resp. extremality). This paper actually extends the results of [14] by semi-discretizing the problem and studying the behavior in the limit and then by applying an optimization method to this discretized problem.

The paper is organized as follows. In section 2 the relaxed controls are introduced, and the classical and the relaxed optimal control problems are formulated. The existence of optimal relaxed controls is also proved. In section 3 the relaxed problem is discretized and in section 4 the behavior in the limit of discrete relaxed optimality and extremality is studied. A penalized conditional descent method is presented in section 5. Two numerical examples are given in section 6.

\section{The continuous optimal control problems}

Let $\Omega$ be a bounded domain in $\mathbb{R}^{d}$, with boundary $\Gamma$, and set $Q:=\Omega \times(0, T), \Sigma:=\Gamma \times(0, T)$ with given final time $T>0$. Consider the following semilinear parabolic initial boundary value problem

$$
\begin{gathered}
y_{t}+A(t) y+\sum_{i=1}^{d} a_{0 i}(x, t) \partial y / \partial x_{i}+b(x, t, y(x, t), u(x, t))=f(x, t, y(x, t), u(x, t)) \text { in } Q, \\
y(x, t)=0 \text { on } \Sigma \\
y(x, 0)=y^{0}(x) \text { in } \Omega .
\end{gathered}
$$

Here $A(t)$ is the elliptic differential operator

$$
A(t) y:=-\sum_{j, i=1}^{d}\left(\partial / \partial x_{i}\right)\left[a_{i j}(x, t) \partial y / \partial x_{j}\right] .
$$

Throughout the paper, we shall use the notation $(\cdot, \cdot),(\cdot, \cdot)_{1},(\cdot, \cdot)_{Q}$ for the inner product and $\|\cdot\|,\|\cdot\|_{1},\|\cdot\|_{Q}$ for the norm of the spaces $L^{2}(\Omega), V:=H_{0}^{1}(\Omega), L^{2}(Q)$ respectively. We define on $V \times V$ the bilinear form associated with $A(t)$

$$
a(t, y, v):=\sum_{j, i=1}^{d} \int_{\Omega} a_{i j}(x, t) \frac{\partial y}{\partial x_{i}} \frac{\partial v}{\partial x_{j}} d x .
$$

Also, $q_{1}$ and $q_{2}$ are given nonnegative integers.

The set of classical controls is defined by

$$
\mathbb{U}:=\{u: Q \rightarrow U \mid u \text { measurable }\} \subset L^{\infty}(Q),
$$

$U \subset \mathbb{R}$ is compact, not necessarily convex and the functionals by

$$
J_{m}(u):=\int_{Q} g_{m}(x, t, y, \nabla y, u) d x d t, \quad m=0, \ldots, q_{2} .
$$

The continuous classical optimal control problem is

$$
\operatorname{minimize} J_{0}(u)
$$

subject to the state equation $(2.1-2.3)$, the control constraints $u \in \mathbb{U}$ and the state constraints

$$
\begin{aligned}
& J_{m}(u)=0, \quad m=1, \ldots, q_{1}, \\
& J_{m}(u) \leq 0, \quad m=q_{1}+1, \ldots, q_{2} .
\end{aligned}
$$


The above problem in order to have a solution has to be endowed with undesirable convexity assumptions (for example, Cesari property), which are usually not realistic for nonlinear systems. But when we formulate the problem in its relaxed form using relaxed controls then the new problem has a solution in a larger space under weaker assumptions.

Let $C(U)$ be the set of continuous functions on $U$ and $M(U)$ (resp. $M_{1}(U)$ ) the set of Radon (resp. probability) measures on $U$. We endow $M(U)=C(U)^{*}$ with the weak* topology. We define the set of relaxed controls ([1], [2])

$$
R:=\left\{r: \bar{Q} \rightarrow M_{1}(U) \mid r \text { weakly measurable }\right\} \subset L_{w}^{\infty}(Q ; M(U)) \equiv L^{1}(Q ; C(U))^{*} .
$$

The topology of $R$ is the weak* topology induced by $L^{1}(Q ; C(U))^{*} . R$ is convex, and with the above topology metrizable and compact. We identify each element $u \in \mathbb{U}$ with the relaxed control $r(\cdot)=\delta_{u(\cdot)}$, where $\delta_{u(\cdot)}$ denotes the Dirac measure concentrated at $u(\cdot)$ and thus we can regard $\mathbb{U}$ as a subset of $R$. Furthermore $\mathbb{U}$ is dense in $R$. For simplicity reasons, for $h \in L^{1}(Q ; C(U))$ and $r \in R$,we write

$$
h(x, t, r(x, t)):=\int_{U} h(x, t, u) r(x, t)(d u)
$$

It follows (see [1]) that $h(x, t, r(x, t))$ is linear in $r$. Let $\left(r_{k}\right)$ be a sequence of relaxed controls and $r \in R$. Then, $\left(r_{k}\right)$ is said to converge to $r$ if and only if

$$
\lim _{k \rightarrow \infty} \int_{Q} h\left(x, t, r_{k}(x, t)\right) d x d t=\int_{Q} h(x, t, r(x, t)) d x d t
$$

for all $h \in L^{1}(Q ; C(U))$.

For the case of noncompact $U$, Fattorini in [3], gives a new definition of relaxed controls based on finitely additive measures on $U$.

The weak relaxed form of the state equation (2.1), using the notation (2.5), is given by

$$
<y_{t}, v>+a(t, y, v)+\sum_{i=1}^{d}\left(a_{0 i}(t) \partial y / \partial x_{i}, v\right)+(b(t, y, r), v)=(f(t, y, r), v)
$$

for every $v \in V$, a.e. in $(0, T), y(t) \in V$, a.e. in $(0, T)$

$$
y(0)=y^{0},
$$

where $\left\langle\cdot, \cdot>\right.$ denotes the dual pairing between $V$ and its dual space $V^{*}=H^{-1}(\Omega)$ and $a(t, y, v)$ is the bilinear form given in (2.4) .

The continuous relaxed optimal control problem (ROCP) is

$$
\operatorname{minimize} J_{0}(r)
$$

subject to the relaxed state equation (2.6), (2.7), the control constraints $r \in R$ and the state constraints

$$
\begin{aligned}
& J_{m}(r)=0, \quad m=1, \ldots, q_{1}, \\
& J_{m}(r) \leq 0, \quad m=q_{1}+1, \ldots, q_{2},
\end{aligned}
$$

where

$$
J_{m}(r):=\int_{Q} \int_{U} g_{m}(x, t, y, \nabla y, u) r(d u) d x d t, \quad m=0, \ldots, q_{2}
$$

We introduce the following assumptions.

(H1) $\Omega \subset \mathbb{R}^{d}, d \leq 3$, is a bounded domain with $C^{1}$-boundary $\Gamma$.

(H2) The coefficient functions $a_{i j}$ of $A(t)$ belong to $L^{\infty}(Q)$ and

$$
\sum_{j=1}^{d} \sum_{i=1}^{d} a_{i j}(x, t) \xi_{i} \xi_{j} \geq \gamma_{0} \sum_{i=1}^{d} \xi_{i}^{2}, \quad \forall \xi_{i}, \xi_{j} \in \mathbb{R},(x, t) \in Q, \text { with } \gamma_{0}>0,
$$

from which easily follow the inequalities

$$
|a(t, y, v)| \leq \alpha_{1}\|y\|_{1}\|v\|_{1}, \quad a(t, v, v) \geq \alpha_{2}\|v\|_{1}^{2}, \quad \forall y, v \in V, \quad t \in(0, T),
$$

for some $\alpha_{1} \geq 0, \alpha_{2}>0$. 
(H3) $a_{0}=\left(a_{01}, \ldots, a_{0 d}\right)^{T} \in L^{\infty}(Q)^{d}$. The functions $b$ and $f: Q \times \mathbb{R} \times U \rightarrow \mathbb{R}$ are measurable w.r.t. $(x, t) \in Q$ for any fixed $y, u$, continuous for fixed $(x, t) \in Q$ and satisfy the conditions

$$
\begin{aligned}
& |b(x, t, y, u)| \leq \varphi(x, t)+\beta|y|^{2}, \quad(x, t, y, u) \in Q \times \mathbb{R} \times U, \\
& |f(x, t, y, u)| \leq \psi(x, t)+\gamma|y|, \quad(x, t, y, u) \in Q \times \mathbb{R} \times U, \\
& \left|f\left(x, t, y_{1}, u\right)-f\left(x, t, y_{2}, u\right)\right| \leq L\left|y_{1}-y_{2}\right|, \quad\left(x, t, y_{1}, y_{2}, u\right) \in Q \times \mathbb{R}^{2} \times U,
\end{aligned}
$$

where $\varphi, \psi \in L^{2}(Q), \beta, \gamma, L \geq 0$.

The function $b$ is monotone increasing with respect to $y$ for almost every $(x, t) \in Q$. Assuming that $b(\cdot, \cdot, y, \cdot)=0$, (if not, we subtract this term from both sides of (2.1)) it follows that $b(x, t, y, u) y \geq 0$.

(H4) The functions $g_{m}: Q \times \mathbb{R}^{d+1} \times U \rightarrow \mathbb{R}$ are measurable for fixed $(y, \bar{y}, u) \in \mathbb{R}^{d+1} \times U$, continuous for fixed $(x, t) \in Q$ and satisfy

$$
\left|g_{m}(x, t, y, \bar{y}, u)\right| \leq \zeta_{m}(x, t)+\delta_{m} y^{2}+\bar{\delta}_{m}|\bar{y}|^{2}, \quad(x, t, y, \bar{y}, u) \in Q \times \mathbb{R}^{d+1} \times U,
$$

with $\zeta_{m} \in L^{1}(Q), \delta_{m} \geq 0, \bar{\delta}_{m} \geq 0$.

(H5) The functions $b_{y}, f_{y}: Q \times \mathbb{R} \times U \rightarrow \mathbb{R}$ are measurable on $Q$ for fixed $(y, u) \in \mathbb{R} \times U$ and continuous on $\mathbb{R} \times U$ for fixed $(x, t) \in Q$ and satisfy

$$
\begin{aligned}
& \left|b_{y}(x, t, y, u)\right| \leq \xi(x, t)+\eta|y|, \quad(x, t, y, u) \in Q \times \mathbb{R} \times U \\
& \left|f_{y}(x, t, y, u)\right| \leq L_{1}, \quad(x, t, y, u) \in Q \times \mathbb{R} \times U
\end{aligned}
$$

with $\xi \in L^{2}(Q), \eta \geq 0, L_{1} \geq 0$.

(H6) The functions $g_{m y}, g_{m \bar{y}}: Q \times \mathbb{R}^{d+1} \times U \rightarrow \mathbb{R}$ are measurable on $Q$ for fixed $(y, \bar{y}, u) \in \mathbb{R}^{d+1} \times U$ and continuous on $\mathbb{R}^{d+1} \times U$ for fixed $(x, t) \in Q$ and satisfy

$$
\begin{aligned}
\left|g_{m y}(x, t, y, \bar{y}, u)\right| & \leq \zeta_{m 1}(x, t)+\delta_{m 1}|y|+\bar{\delta}_{m 1}|\bar{y}|^{2}, \quad(x, t, y, \bar{y}, u) \in Q \times \mathbb{R}^{d+1} \times U, \\
\left|g_{m \bar{y}}(x, t, y, \bar{y}, u)\right| & \leq \zeta_{m 2}(x, t)+\delta_{m 2} y^{2}+\bar{\delta}_{m 2}|\bar{y}|, \quad(x, t, y, \bar{y}, u) \in Q \times \mathbb{R}^{d+1} \times U
\end{aligned}
$$

with $\zeta_{m 1}, \zeta_{m 2} \in L^{2}(Q), \delta_{m 1}, \bar{\delta}_{m 1}, \delta_{m 2}, \bar{\delta}_{m 2} \geq 0$

Using assumptions (H1-H3) and the fact that $V$ is compactly embedded in $L^{4}(\Omega)$, we can see that equation (2.6) is well defined.

Theorem 2.1. Under Assumptions (H1-H3), for every $r \in R$ and $y^{0} \in L^{2}(\Omega)$ (or $\left.y^{0} \in V\right)$, there exist a unique $y:=y_{r}$ such that $y \in L^{2}((0, T), V), y_{t} \in L^{2}\left((0, T), V^{*}\right)$ satisfying (2.6), (2.7). In addition, $y$ is essentially equal to a function in $C\left([0, T], L^{2}(\Omega)\right)$, and thus the initial condition (2.7) is well defined.

Proof. The proof is based on compactness arguments (see [15]).

Next lemma describes the continuity of the state and the functionals w.r.t. the corresponding relaxed control. This result is the basic tool to prove the existence of optimal relaxed controls.

Lemma 2.2. Under Assumptions (H1-H3), the mapping $r \mapsto y_{r}$, from $R$ to $L^{2}(Q)$ and $L^{2}((0, T), V)$, is continuous. Under Assumptions (H1-H4), the functionals $r \mapsto J_{m}(r), m=0, \ldots, q_{2}$, from $R$ to $\mathbb{R}$, are continuous.

Proof. Let $r_{k} \rightarrow r$ in $R$ and set $y_{k}:=y_{r_{k}}$. Taking $y=v=y_{k}$ in (2.6), using Assumptions (H2-H3) and the basic inequality $2 a b \leq \frac{1}{\varepsilon} a^{2}+\varepsilon b^{2}, \varepsilon>0$, we have

$$
\frac{1}{2} \frac{d}{d t}\left\|y_{k}\right\|^{2}+a_{2}\left\|y_{k}\right\|_{1}^{2} \leq \frac{1}{2}\|\psi(t)\|^{2}+\left(\frac{1}{2}+\gamma+\frac{c}{2 \varepsilon}\right)\left\|y_{k}\right\|^{2}+c \frac{\varepsilon}{2}\left\|y_{k}\right\|_{1}^{2}, \text { where } c:=\left\|a_{0}\right\|_{L^{\infty}} .
$$

Integrating w.r.t. $t$ on $[0, t]$ for $t \leq T$ and selecting appropriate $\varepsilon>0$ to hide the term $\int_{0}^{t}\left\|y_{k}(s)\right\|_{1}^{2} d s$ to the left-hand side we obtain

$$
\frac{1}{2}\left\|y_{k}\right\|^{2}+\left(a_{2}-c \frac{\varepsilon}{2}\right) \int_{0}^{t}\left\|y_{k}(s)\right\|_{1}^{2} d s \leq \frac{1}{2}\left\|y^{0}\right\|^{2}+\frac{1}{2}\|\psi\|_{L^{2}(Q)}^{2}+\left(\frac{1}{2}+\gamma+\frac{c}{2 \varepsilon}\right) \int_{0}^{t}\left\|y_{k}(s)\right\|^{2} d s .
$$


Using Gronwall's inequality we deduce from (2.8) that $y_{k}$ is bounded in $L^{2}(Q)$. Then again from (2.8) we obtain that $y_{k}$ is bounded in $L^{2}((0, T), V)$. One can also check using (2.6) that $y_{k}^{\prime}$ is bounded in $L^{2}\left((0, T), V^{*}\right)$. Thus, there exist a subsequence still denoted by $\left(y_{k}\right)$ such that $y_{k} \longrightarrow y$ in $L^{2}((0, T), V)$ weakly and $y_{k}^{\prime} \longrightarrow y^{\prime}$ in $L^{2}\left((0, T), V^{*}\right)$ weakly. Since $V$ is compactly embedded in $L^{2}(\Omega)$ by Theorem 2.1 chap. III in [16] follows that $y_{k} \longrightarrow y$ in $L^{2}(Q)$ strongly. It follows easily that $y=y_{r}$ and that the convergence holds for the original sequence. The strong convergence $y_{k} \longrightarrow y$ in $L^{2}((0, T), V)$ can be proved as in Lemma 4.2 here. Finally, from Proposition 2.1 in [6] we derive that the functionals $r \mapsto J_{m}(r), m=0, \ldots, q_{2}$ are continuous.

Theorem 2.3. Under Assumptions (H1-H4) and supposing the existence of a feasible control the ROCP has a solution.

Proof. It follows from Lemma 2.2 and the compactness of $R$.

Necessary conditions for optimality for the ROCP are given in Chryssoverghi et al. [14].

\section{The semi-discrete optimal control problems}

(H7) $a, a_{0}$ are independent of $t$ (for simplicity), $b, b_{y}, f, f_{y}$ are continuous on $\bar{Q} \times \mathbb{R} \times U, g_{m}, g_{m y}, g_{m \bar{y}}$ are continuous on $\bar{Q} \times \mathbb{R}^{d+1} \times U$ and $y^{0} \in V$.

For each integer $n \geq 0$, let $\Omega^{n}$ be a subdomain of $\Omega$ with polyhedral boundary $\Gamma^{n}$ such that $\operatorname{dist}\left(\Gamma^{n}, \Gamma\right)=o\left(h^{n}\right),\left\{E_{i}^{n}\right\}_{i=1}^{M^{n}}$ be an admissible regular quasi-uniform triangulation of $\bar{\Omega}^{n}$ into closed $d$-simplices (finite elements), with $h^{n}=\max _{i}\left[\operatorname{diam}\left(E_{i}^{n}\right)\right] \rightarrow 0$ as $n \rightarrow \infty$. Associated with the above triangulation we define

$$
V^{n}:=\left\{y^{n} \in V \mid y^{n} \in C(\bar{\Omega}), \text { affine on each } E_{i}^{n}, y^{n}=0 \text { in } \Omega-\Omega^{n}\right\}
$$

with $\operatorname{dim} V^{n}=N^{n}$ and $v_{i}^{n}, i=1, \ldots, N^{n}$ be a basis of $V^{n}$,

$$
R^{n}:=\left\{r^{n} \in R \mid r^{n}=\left\{r_{i}^{n}, i=1, \ldots M^{n}\right\}, r_{i}^{n} \text { is equal to a constant measure w.r.t. } x \text { in } M_{1}(U) \text { on the interior of }\left(E_{i}^{n} \times(0, T)\right), i=1, \ldots M^{n}\right\}
$$

the set of (semi)discrete relaxed controls and $\mathbb{U}^{n}:=R^{n} \cap U$ the set of (semi)discrete classical controls. Clearly, we have $\mathbb{U}^{n} \subset R^{n}$.

For a given $r^{n} \in R^{n}$, the corresponding (semi)discrete state $y^{n}$ is given by the (semi)discrete state equation (system of ODE's w.r.t. $\left.c^{n}\right)$

$$
\begin{gathered}
\left(y^{n^{\prime}}, v_{i}^{n}\right)+a\left(y^{n}, v_{i}^{n}\right)+\left(a_{0}^{T}(t) \nabla y^{n}, v_{i}^{n}\right)+\left(b\left(t, y^{n}, r^{n}\right), v_{i}^{n}\right)=\left(f\left(t, y^{n}, r^{n}\right), v_{i}^{n}\right), i=1, \ldots, N^{n}, \forall t \in(0, T), \\
\left(y^{n}(0)-y^{0}, v_{i}^{n}\right)_{1}=0, i=1, \ldots, N^{n},
\end{gathered}
$$

where $y^{n}(t)=\sum_{i=1}^{N^{n}} c_{i}^{n}(t) v_{i}^{n}$. Note that $y^{n}(0)$ is the orthogonal projection of $y^{0}$ onto $V$.

Theorem 3.1. Under Assumptions (H2-H3) and (H7), for every $n$ and $r^{n} \in R^{n}$ the equation (3.1), (3.2) admits a unique solution $y^{n}$. In addition, the solutions are uniformly (w.r.t. $\left.r^{n}\right)$ bounded and equicontinuous.

The discrete functionals are defined by

$$
J_{m}^{n}\left(r^{n}\right):=\int_{Q} g_{m}\left(x, t, y^{n}, \nabla y^{n}, r^{n}\right) d x d t, \quad m=0, \ldots, q_{2} .
$$

We consider the following two discrete problems:

$$
\operatorname{minimize} J_{0}^{n}\left(r^{n}\right)
$$

subject to (3.1), (3.2), the control constraints $r^{n} \in R^{n}$ and the state constraints

$$
\begin{array}{ll}
\text { Case (a) } \quad & \left|J_{m}^{n}\left(r^{n}\right)\right| \leq \varepsilon_{m}^{n}, \quad m=1, \ldots, q_{1} \\
& J_{m}^{n}\left(r^{n}\right) \leq \varepsilon_{m}^{n}, \quad \varepsilon_{m}^{n} \geq 0, \quad m=q_{1}+1, \ldots, q_{2},
\end{array}
$$

and

$$
\begin{array}{ll}
\text { Case (b) } \quad & J_{m}^{n}\left(r^{n}\right)=\varepsilon_{m}^{n}, \quad m=1, \ldots, q_{1}, \\
& J_{m}^{n}\left(r^{n}\right) \leq \varepsilon_{m}^{n}, \quad \varepsilon_{m}^{n} \geq 0, \quad m=q_{1}+1, \ldots, q_{2},
\end{array}
$$

where $\varepsilon_{m}^{n}$ are non-negative given numbers, introduced for feasibility reasons.

The first of the above discrete problems with state constraints (3.3) is denoted by DROCP $P_{a}$ and the second one with state constraints (3.4) by $\mathrm{DROCP}_{b}$. 
Theorem 3.2. Under Assumptions (H2-H4) and (H7), the mappings $r^{n} \mapsto y^{n}$ and $r^{n} \mapsto J_{m}^{n}\left(r^{n}\right)$, defined on $R^{n}$, are continuous. If any of the discrete problems is feasible, then it has a solution.

Proof. The continuity of the operator $r^{n} \mapsto y^{n}$ is proved by Theorem 3.1 and using Ascoli's theorem to pass in the limit in (3.1), (3.2). The continuity of $r^{n} \rightarrow J_{m}^{n}\left(r^{n}\right)$ follows from the continuity of $g_{m}$. Since the set $R^{n}$ is compact with the relative weak* topology of $M(U)^{M^{n}}$ it follows that the discrete problems $D R O C P_{a}, D R O C P_{b}$ defined above have a solution.

To compute the directional derivative of the functional $J^{n}$, where for simplicity reasons the index $m$ is omitted, we introduce the linear adjoint state equation

$$
\begin{gathered}
-\left(z^{n^{\prime}}, v\right)+a\left(v, z^{n}\right)+\left(a_{0}^{T} \nabla v, z^{n}\right)+\left(z^{n} b_{y}\left(t, y^{n}, r^{n}\right), v\right)=\left(z^{n} f_{y}\left(t, y^{n}, r^{n}\right)+g_{y}\left(t, y^{n}, \nabla y^{n}, r^{n}\right), v\right)+\left(g_{\bar{y}}\left(t, y^{n}, \nabla y^{n}, r^{n}\right), \nabla v\right), \forall v \in V^{n}, \\
z^{n}(T)=0,
\end{gathered}
$$

which has a unique solution $z^{n}=z_{r^{n}}$ with $y^{n}=y_{r^{n}}$.

We define, for each function $g$, the Hamiltonian $H$

$$
H(x, t, y, \bar{y}, z, u):=z[f(x, t, y, u)-b(x, t, y, u)]+g(x, t, y, \bar{y}, u) .
$$

The following lemma and theorem can be proved by using the techniques of [1], [6]. See also [17], where necessary optimality conditions on signomial constrained optimal control problems are proved.

Lemma 3.3. Under Assumptions (H2-H7), the directional derivative of the functional $\mathrm{J}^{n}$ is given by

$$
D J^{n}\left(r^{n}, r^{\prime n}-r^{n}\right)=\int_{Q} H\left(x, t, y^{n}, \nabla y^{n}, z^{n}, r^{\prime n}-r^{n}\right) d x d t, r^{n}, r^{\prime n} \in R^{n},
$$

where $z^{n}$ is given by (3.5), (3.6). Moreover, the mappings $r^{n} \mapsto z^{n}$ and $\left(r^{n}, r^{\prime n}\right) \mapsto D J^{n}\left(r^{n}, r^{\prime n}-r^{n}\right)$ are continuous.

Proof. For simplicity of notation we drop the index $n$. For $r, r^{\prime} \in R, 0<\varepsilon \leq 1$, set $r_{\varepsilon}=r+\varepsilon\left(r^{\prime}-r\right), y:=y_{r}, y_{\varepsilon}:=y_{r_{\varepsilon}}$, $\delta_{\varepsilon} y:=y_{\varepsilon}-y$. Now, by our assumptions, for fixed $r \in R$, the functional

$$
\Phi(y, \bar{y}, r):=\int_{Q} g(x, t, y, \bar{y}, r) d x d t,
$$

is Fréchet differentiable uniformly in $r$, i.e.

$$
\Phi(y+\delta y, \bar{y}+\delta \bar{y}, r)-\Phi(y, \bar{y}, r)=\int_{Q}\left[g_{y}(x, t, y, \bar{y}, r) \delta y+g_{\bar{y}}(x, t, y, \bar{y}, r) \delta \bar{y}\right] d x d t+\theta(\delta y, \delta \bar{y})\left(\|\delta y\|_{\infty}+\|\delta \bar{y}\|\right),
$$

where $\theta(\delta y, \delta \bar{y}) \rightarrow 0$ as $\|\delta y\|_{\infty}+\|\delta \bar{y}\| \rightarrow 0$, with $\theta$ independent of the control $r \in R$. This can be shown under our assumptions by using the Mean Value Theorem, Hölder's inequality and Proposition 2.1 in [6] for a fixed control. By Lemma 2.2 in [6], we have

$$
\begin{aligned}
J\left(r_{\varepsilon}\right)-J(r) & =\int_{Q}\left[g\left(y_{\varepsilon}, \nabla y_{\varepsilon}, r_{\varepsilon}\right)-g\left(y, \nabla y, r_{\varepsilon}\right)+g\left(y, \nabla y, r_{\varepsilon}\right)-g(y, \nabla y, r)\right] d x d t \\
& =\int_{Q} g_{y}(y, \nabla y, r) \delta_{\varepsilon} y d x d t+\int_{Q} g_{\bar{y}}(y, \nabla y, r) \nabla \delta_{\varepsilon} y d x d t+\varepsilon \int_{Q} g\left(y, \nabla y, r^{\prime}-r\right) d x d t+o(\varepsilon) .
\end{aligned}
$$

Since $\delta_{\varepsilon} y(0)=z(T)=0$, by similar arguments, the state equation (3.1) yields

$$
-\int_{0}^{T}\left(z^{\prime}, \delta_{\varepsilon} y\right) d t+\int_{0}^{T} a\left(\delta_{\varepsilon} y, z\right) d t+\int_{0}^{T}\left(a_{0}^{T} \nabla \delta_{\varepsilon} y, z\right) d t=\int_{Q}\left(f_{y}(y, r)-b_{y}(y, r)\right) \delta_{\varepsilon} y z d x d t+\varepsilon \int_{Q}\left(f\left(y, r^{\prime}-r\right)-b\left(y, r^{\prime}-r\right)\right) z d x d t+o(\varepsilon) .
$$

On the other hand, the adjoint equation (3.5) yields

$$
\begin{aligned}
-\int_{0}^{T}\left(z^{\prime}, \delta_{\varepsilon} y\right) d t & +\int_{0}^{\mathrm{T}} a\left(\delta_{\varepsilon} y, z\right) d t+\int_{0}^{T}\left(a_{0}^{T} \nabla \delta_{\varepsilon} y, z\right) d t \\
& =\int_{Q}\left(f_{y}(y, r)-b_{y}(y, r)\right) \delta_{\varepsilon} y z d x d t+\int_{Q} g_{y}(y, \nabla y, r) \delta_{\varepsilon} y d x d t+\int_{Q} g_{\bar{y}}(y, \nabla y, r) \nabla \delta_{\varepsilon} y d x d t .
\end{aligned}
$$

Gathering (3.7), (3.8) and (3.9), we obtain

$$
D J\left(r, r^{\prime}-r\right)=\int_{Q}\left[z\left(f\left(x, t, y, r^{\prime}-r\right)-b\left(x, t, y, r^{\prime}-r\right)\right)+g\left(x, t, y, \nabla y, r^{\prime}-r\right)\right] d x d t .
$$


Theorem 3.4. (i) Under Assumptions (H2-H7), if $r^{n} \in R^{n}$ is a solution of the DROCP , then it is extremal, i.e. there exist multipliers $\lambda_{m}^{n} \in \mathbb{R}, m=0, \ldots, q_{2}$, with $\lambda_{0}^{n} \geq 0, \lambda_{m}^{n} \geq 0, m=q_{1}+1, \ldots, q_{2}, \sum_{m=0}^{q_{2}}\left|\lambda_{m}^{n}\right|=1$, such that

$$
\begin{gathered}
\sum_{m=0}^{q_{2}} \lambda_{m}^{n} D J_{m}^{n}\left(r^{n}, r^{\prime n}-r^{n}\right)=\int_{Q} H\left(x, t, y^{n}, \nabla y^{n}, z^{n}, r^{\prime n}-r^{n}\right) d x d t \geq 0, \forall r^{\prime n} \in R^{n} \\
\lambda_{m}^{n}\left[J_{m}^{n}\left(r^{n}\right)-\varepsilon_{m}^{n}\right]=0, \quad m=q_{1}+1, \ldots, q_{2}
\end{gathered}
$$

where $H$ and $z^{n}$ are defined with $g:=\sum_{m=0}^{q_{2}} \lambda_{m}^{n} g_{m}$. Condition (3.10) is equivalent to the strong discrete block pointwise minimum principle

$$
\int_{E_{i}^{n}} H\left(x, t, y^{n}, \nabla y^{n}, z^{n}, r^{n}\right) d x=\min _{u \in U} \int_{E_{i}^{n}} H\left(x, t, y^{n}, \nabla y^{n}, z^{n}, u\right) d x, i=1, \ldots, M^{n} \text {, a.e.in }(0, T) .
$$

(ii) With Assumptions (H2-H7) and assuming that $J_{0}^{n}, J_{q_{1}+1}^{n}, \ldots, J_{q_{2}}^{n}$ are convex and $J_{1}^{n}, \ldots, J_{q_{1}}^{n}$ are affine, if $r^{n} \in R^{n}$ is admissible and extremal for the $\mathrm{DROCP}_{b}$, with $\lambda_{0}^{n}>0$, then $r^{n}$ is optimal for this problem.

Proof. (i) The global condition (3.10) and the conditions (3.11) follow from the general multiplier theorem V.2.3 in [1]. The equivalence of the conditions (3.10) and (3.12) is standard (see [1]), since the closed set $U$ has a dense denumerable subset.

(ii) The assumptions imply that the functional $J^{n}\left(r^{n}\right):=\sum_{m=0}^{q} \lambda_{m}^{n} J_{m}^{n}\left(r^{n}\right)$ is convex. The condition (3.10) is then satisfied if and only if $r^{n}$ minimizes $J^{n}$ on $R^{n}$. Supposing now that $r^{n}$ does not minimize $J_{0}^{n}$ and using the constraints and the conditions (3.11), easily follows that $r^{n}$ does not minimize $J^{n}$, which is a contradiction.

\section{Behavior in the limit}

Here we study the limiting behavior of the discrete problems as $n \rightarrow \infty$. Next proposition gives us a control approximation result. It is proved in [6] for totally (i.e. in space and time) discrete controls, from which it follows for semidiscrete ones.

Proposition 4.1. For every $r \in R$, there exist $\left(u^{n}\right) \in \mathbb{U}^{n}$ such that $u^{n} \rightarrow r$ in $R$.

Lemma 4.2 (Consistency of states and functionals). Under Assumptions (H2-H3) and (H7), if $r^{n} \rightarrow r$ in $R$, then the corresponding discrete states $y^{n}$ converge to $y_{r}$ in $L^{2}((0, T), V)$ strongly and

$$
\lim _{n \rightarrow \infty} J_{m}^{n}\left(r^{n}\right)=J_{m}(r), \quad m=0, \ldots, q_{2}
$$

Proof. Multiplying (3.1) by $c_{i}^{n}$ and summing over $i$ we obtain for every $t \in(0, T)$

$$
\left\langle y^{n^{\prime}}, y^{n}\right\rangle+a\left(y^{n}, y^{n}\right)+\left(a_{0}^{T}(t) \nabla y^{n}, y^{n}\right)+\left(b\left(t, y^{n}, r^{n}\right), y^{n}\right)=\left(f\left(t, y^{n}, r^{n}\right), y^{n}\right) .
$$

Integrating (4.2) on $[0, t], t \leq T$ and working similarly to the proof of Lemma 2.2, we deduce that

$$
\frac{1}{2}\left\|y^{n}(t)\right\|^{2}+c_{1} \int_{0}^{t}\left\|y^{n}(s)\right\|_{1}^{2} d s \leq \frac{1}{2}\left\|y^{n}(0)\right\|^{2}+\frac{1}{2} \int_{0}^{t}\|\psi(s)\|^{2} d s+c_{2} \int_{0}^{t}\left\|y^{n}(s)\right\|^{2} d s, \forall t \in(0, T),
$$

for some appropriate constants $c_{1}, c_{2}$.

Using Gronwall's inequality and the fact that $y^{n}(0)$ is bounded (since clearly $y^{n}(0) \rightarrow y^{n}$ in $V$ strongly, due to the projection) we deduce from (4.3) that $y^{n}$ is bounded in $L^{2}(Q)$. Then from (4.3) we obtain that $y^{n}$ is bounded in $L^{2}((0, T), V)$. One can also prove using (3.1) that $y^{n^{\prime}}$ is bounded in $L^{2}\left((0, T), V^{*}\right)$. Since the injection of $V$ in $L^{2}(\Omega)$ is compact, by the compactness Theorem 2.2 chap. III in [16], there exists a subsequence still denoted by $y^{n}: y^{n} \rightarrow y$ in $L^{2}(Q)$ strongly.

Let $v \in C_{0}^{1}(\bar{\Omega})$ an arbitrary given function and $\left(v^{n}\right) \in V^{n}$ a sequence of functions interpolating the function $v$ at the vertices inside $\Omega^{n}$ and vanishing on $\Gamma^{n}$. The sequence converges to $v$ in $V$ strongly. Then, the integral form of the discrete equation (3.1) is written

$$
\left(y^{n}(T), v^{n}\right)-\left(y_{0}^{n}, v^{n}\right)+\int_{0}^{T} a\left(y^{n}, v^{n}\right) d t+\int_{0}^{T}\left(a_{0}^{T} \nabla y^{n}, v^{n}\right) d t=\int_{0}^{T}\left(f\left(t, y^{n}, r^{n}\right)-b\left(t, y^{n}, r^{n}\right), v^{n}\right) d t .
$$


We obtain from (4.4) using the above convergences and Proposition 2.1 in [6] that, $\forall v \in C_{0}^{1}(\bar{\Omega})$

$$
\begin{aligned}
& \left(y^{n}(T), v\right)=\left(y^{n}(T), v-v^{n}\right)+\left(y^{n}(T), v^{n}\right)=\left(y^{n}(T), v-v^{n}\right)+\left(y_{0}^{n}, v^{n}\right) \\
& +\int_{0}^{T}\left(f\left(t, y^{n}, r^{n}\right)-b\left(t, y^{n}, r^{n}\right), v^{n}\right) d t-\int_{0}^{T} a\left(y^{n}, v^{n}\right) d t-\int_{0}^{T}\left(a_{0}^{T} \nabla y^{n}, v^{n}\right) d t \\
& \longrightarrow\left(y^{0}, v\right)+\int_{0}^{T}(f(y, r)-b(y, r), v) d t-\int_{0}^{T} a(y, v) d t-\int_{0}^{T}\left(a_{0}^{T} \nabla y, v\right) d t=(y(T), v) .
\end{aligned}
$$

Since $C_{0}^{1}(\bar{\Omega})$ is dense in $L^{2}(\Omega)$ it follows that $\left(y^{n}(T), v\right) \rightarrow(y(T), v) \forall v \in L^{2}(\Omega)$, i.e. $y^{n}(T) \rightarrow y(T)$ in $L^{2}(\Omega)$ weakly. By the above convergences, we get from (4.4)

$$
(y(T), v)-\left(y^{0}, v\right)+\int_{0}^{T} a(y, v) d t+\int_{0}^{T}\left(a_{0}^{T} \nabla y, v\right) d t=\int_{0}^{T}(f(y, r)-b(y, r), v) d t,
$$

hence $y=y_{r}$.

Next, we prove that $y^{n} \rightarrow y$ in $L^{2}((0, T), V)$ strongly. We have

$$
\begin{gathered}
\alpha_{2}\left\|y^{n}-y\right\|_{L^{2}((0, T), V)}^{2} \leq \int_{0}^{T} a\left(y^{n}-y, y^{n}-y\right) d t+\frac{1}{2}\left\|y^{n}(T)-y(T)\right\|^{2}=\frac{1}{2}\left\|y_{0}^{n}\right\|^{2}-\frac{1}{2}\left(y^{n}(T), y(T)\right)-\frac{1}{2}\left(y(T), y^{n}(T)-y(T)\right) \\
+\int_{0}^{T}\left(f\left(y^{n}, r^{n}\right)-b\left(y^{n}, r^{n}\right), y^{n}\right) d t-\int_{0}^{T}\left(a_{0}^{T} \nabla y^{n}, y^{n}\right) d t-\int_{0}^{T} a\left(y^{n}, y\right) d t-\int_{0}^{T} a\left(y, y^{n}-y\right) d t
\end{gathered}
$$

and as $n \rightarrow \infty$ the right-hand side of the above inequality convergence to zero.

Finally, the convergences (4.1) follow from Proposition 2.1 in [6].

In what follows the feasibility of the ROCP is assumed. Next theorem addresses the limit behavior of optimal discrete relaxed controls for the $\mathrm{DROCP}_{a}$.

Theorem 4.3. Under Assumptions $(H 2-H 4)$ and $(H 7)$ and the additional assumption that the sequences $\left(\varepsilon_{m}^{n}\right)$ converge to zero as $n \rightarrow \infty$ and satisfy

$$
\left|J_{m}^{n}\left(\tilde{r}^{n}\right)\right| \leq \varepsilon_{m}^{n}, \quad m=1, \ldots, q_{1}, \quad J_{m}^{n}\left(\tilde{r}^{n}\right) \leq \varepsilon_{m}^{n}, \quad \varepsilon_{m}^{n} \geq 0, \quad m=q_{1}+1, \ldots, q_{2},
$$

for every $n$, where $\left(\tilde{r}^{n}\right) \in R^{n}$ is a sequence which converges in $R$ to some $\tilde{r} \in R$ optimal for the ROCP. Then, for each $n$, we

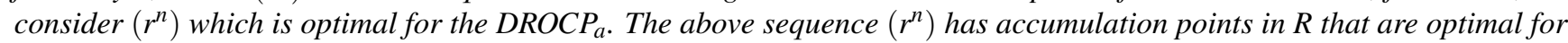
the ROCP.

Proof. From theorem's assumptions the feasibility of the $D R O C P_{a}$, for every $n$, follows. Let a subsequence of $\left(r^{n}\right)$, still denoted by $\left(r^{n}\right)$, such that $r^{n} \rightarrow r, r \in R$. Since $r^{n}$ is admissible as optimal and $\tilde{r}^{n}$ is admissible for the $D R O C P_{a}$, it follows

$$
J_{0}^{n}\left(r^{n}\right) \leq J_{0}^{n}\left(\tilde{r}^{n}\right),\left|J_{m}^{n}\left(r^{n}\right)\right| \leq \varepsilon_{m}^{n}, m=1, \ldots, q_{1}, J_{m}^{n}\left(r^{n}\right) \leq \varepsilon_{m}^{n}, m=q_{1}+1, \ldots, q_{2} .
$$

Taking limits as $n \rightarrow \infty$, with the help of Lemma 4.2, we conclude that $r$ is optimal for the ROCP.

Lemma 4.4. Under Assumptions (H2-H7), if $r^{n} \rightarrow r$ in $R$, then $z^{n} \rightarrow z_{r}$ in $L^{2}((0, T), V)$ strongly, where $z^{n}$ the corresponding discrete adjoint states. If $r^{n} \rightarrow r$ and $r^{\prime n} \rightarrow r^{\prime}$, then

$$
\lim _{n \rightarrow \infty} D J_{m}^{n}\left(r^{n}, r^{\prime n}-r^{n}\right)=D J_{m}\left(r, r^{\prime}-r\right), \quad m=0, \ldots, q_{2}
$$

Proof. It follows easily from Lemma 4.2 and the same arguments as those in the proof of that Lemma.

Next, we consider the $D R O C P_{b}$. We can choose $\left(\varepsilon_{m}^{n}\right), m=1, \ldots, q_{2}$, such that $\varepsilon_{m}^{n} \rightarrow 0, n \rightarrow \infty$ and the $D R O C P_{b}$ is feasible for every $n$ (see [6]).

Theorem 4.5. Under Assumptions (H2-H7), for each $n$, let $r^{n}$ be admissible and extremal for the DROCP. Then the sequence $\left(r^{n}\right)$ has accumulation points that are admissible and extremal for the ROCP.

Proof. Since $R$ is compact, consider a subsequence $\left(r^{n}\right)$ such that $r^{n} \rightarrow r$ in $R$. From Theorem 3.4, there exist multipliers $\lambda_{m}^{n} \in \mathbb{R}, m=0, \ldots, q_{2}$ with $\sum_{m=0}^{q_{2}}\left|\lambda_{m}^{n}\right|=1$, thus there exist subsequences $\left(\lambda_{m}^{n}\right), m=0, \ldots, q_{2}$, such that $\lambda_{m}^{n} \rightarrow \lambda_{m}, m=0, \ldots, q_{2}$. Let any $r^{\prime} \in R$ and $\left(r^{\prime n}\right)$ be a sequence such that $r^{\prime n} \rightarrow r^{\prime}$ (Proposition 4.1). Using the above convergences, Lemmas 4.2, 4.4 and Proposition 2.1 in [6] and passing to the limit in (3.10), (3.11) we have

$$
\int_{Q} H\left(x, t, y, \nabla y, z, r^{\prime}(x, t)-r(x, t)\right) d x d t \geq 0, \quad \forall r^{\prime} \in R
$$




$$
\lambda_{m} J_{m}(r)=\lim _{n \rightarrow \infty} \lambda_{m}^{n}\left[J_{m}^{n}\left(r^{n}\right)-\varepsilon_{m}^{n}\right]=0, \quad m=q_{1}+1, \ldots, q_{2}
$$

Also,

$$
\begin{gathered}
J_{m}(r)=\lim _{n \rightarrow \infty}\left[J_{m}^{n}\left(r^{n}\right)-\varepsilon_{m}^{n}\right]=0, \quad m=1, \ldots, q_{1}, \\
J_{m}(r)=\lim _{n \rightarrow \infty}\left[J_{m}^{n}\left(r^{n}\right)-\varepsilon_{m}^{n}\right] \leq 0, \quad m=q_{1}+1, \ldots, q_{2} .
\end{gathered}
$$

Therefore, $r$ is admissible and extremal for the ROCP (see [14]).

\section{Discrete penalized conditional descent method}

We choose a fixed discretization and for notational simplicity we shall drop the index $n$ in the data. Let $\left(M_{m}^{l}\right), m=1, \ldots, q_{2}$, be increasing sequences with $\left(M_{m}^{l}\right)>0$ and $M_{m}^{l} \rightarrow \infty$ as $l \rightarrow \infty$. Define the discrete functionals with penalties

$$
J^{l}(r):=J_{0}(r)+0.5\left\{\sum_{m=1}^{q_{1}} M_{m}^{l}\left[J_{m}(r)\right]^{2}+\sum_{m=q_{1}+1}^{q_{2}} M_{m}^{l}\left[\max \left(0, J_{m}(r)\right)\right]^{2}\right\} .
$$

Let $\rho, \sigma \in(0,1)$, and let $\left(\beta^{l}\right),\left(\zeta_{k}\right)$ be positive sequences, with $\left(\beta^{l}\right)$ decreasing and converging to zero, and $\zeta_{k} \leq 1$. A penalized conditional descent method with Armijo line step search applied on the $D R O C P_{b}$ is presented in the following algorithm.

\section{Algorithm}

Step 1. $k=0, l=1$. Choose an initial discrete control $r_{0}^{1} \in R$.

Step 2. Compute the state and the adjoint associated with $r_{k}^{l}$. Find $\bar{r}_{k}^{l} \in R$ such that

$$
\bar{r}_{k}^{l}=\arg \min \left\{D J^{l}\left(r_{k}^{l}, r^{\prime}-r_{k}^{l}\right), r^{\prime} \in R\right\}
$$

and set $d_{k}:=D J^{l}\left(r_{k}^{l}, \bar{r}_{k}^{l}-r_{k}^{l}\right)$.

Step 3. If $\left|d_{k}\right|>\beta^{l}$, then go to Step 4, else $r^{l}=r_{k}^{l}, \bar{r}^{l}=\bar{r}_{k}^{l}, d^{l}=d_{k}, r_{k}^{l+1}=r_{k}^{l}, l=l+1$ and return to Step 2.

Step 4. Find the smallest nonnegative integer $s$, denoted $\bar{s}$ :

$$
J^{l}\left(r_{k}^{l}+\sigma^{s} \zeta_{k}\left(\bar{r}_{k}^{l}-r_{k}^{l}\right)\right)-J^{l}\left(r_{k}^{l}\right) \leq \sigma^{s} \zeta_{k} \rho d_{k}
$$

Set $\alpha_{k}=\sigma^{\bar{s}} \zeta_{k}$.

Step 5. Choose an equivalent $r_{k+1}^{l} \in R$ such that

$$
J^{l}\left(r_{k+1}^{l}\right)=J^{l}\left(r_{k}^{l}+\alpha_{k}\left(\bar{r}_{k}^{l}-r_{k}^{l}\right)\right),
$$

set $k=k+1$, and return to Step 2 .

We now define the sequences of multipliers

$$
\lambda_{m}^{l}:=M_{m}^{l} J_{m}\left(r^{l}\right), m=1, \ldots, q_{1}, \lambda_{m}^{l}:=M_{m}^{l} \max \left(0, J_{m}\left(r^{l}\right)\right), m=q_{1}+1, \ldots, q_{2},
$$

where $r^{l}$ are defined in Step 3 of the Algorithm.

In the following theorem we study the convergence properties of the above algorithm.

Theorem 5.1. Consider the sequence $\left(r^{l}\right)$ constructed in Step 3 of the Algorithm. If the sequences $\left(\lambda_{m}^{l}\right), m=1, \ldots, q_{2}$ remain bounded, then any accumulation point of $\left(r^{l}\right)$ satisfies the optimality conditions (3.10), (3.11) for the discrete problem.

Proof. We can prove that $l \rightarrow \infty$ in the Algorithm as in Theorem 5.1 in [12].

If $r \in R$ is an accumulation point of the sequence $\left(r^{l}\right)$, there exist a subsequence of it, still denoted by $\left(r^{l}\right)$, converging to $r \in R$ as $l \rightarrow \infty$. If the sequences $\left(\lambda_{m}^{l}\right), m=1, \ldots, q_{2}$ defined in (5.1) are bounded, then they have subsequences, again denoted by $\left(\lambda_{m}^{l}\right)$, such that $\lambda_{m}^{l} \rightarrow \lambda_{m}$. Using Lemma 4.2, we obtain

$$
0=\lim _{l \rightarrow \infty} \frac{\lambda_{m}^{l}}{M_{m}^{l}}=\lim _{l \rightarrow \infty} J_{m}\left(r^{l}\right)=J_{m}(r), \quad m=1, \ldots, q_{1},
$$




$$
0=\lim _{l \rightarrow \infty} \frac{\lambda_{m}^{l}}{M_{m}^{l}}=\lim _{l \rightarrow \infty}\left[\max \left(0, J_{m}\left(r^{l}\right)\right)\right]=\max \left(0, J_{m}(r)\right), \quad m=q_{1}+1, \ldots, q_{2},
$$

thus $r$ is admissible. Next, for every $r^{\prime} \in R$, Steps 2, 3 of the Algorithm give

$$
D J^{l}\left(r^{l}, r^{\prime}-r^{l}\right)=\lambda_{0}^{l} D J_{0}\left(r^{l}, r^{\prime}-r^{l}\right)+\sum_{m=1}^{q_{1}} \lambda_{m}^{l} D J_{m}\left(r^{l}, r^{\prime}-r^{l}\right)+\sum_{m=q_{1}+1}^{q_{2}} \lambda_{m}^{l} D J_{m}\left(r^{l}, r^{\prime}-r^{l}\right) \geq d^{l},
$$

with $\lambda_{0}^{l}:=1$. From Step 3 of the Algorithm we have $\left|d^{l}\right| \leq \beta^{l} \rightarrow 0$. We use the above convergences and Lemma 3.3 to pass to the limit in (5.2), as $l \rightarrow \infty$ and obtain

$$
\lambda_{0} D J_{0}\left(r, r^{\prime}-r\right)+\sum_{m=1}^{q_{1}} \lambda_{m} D J_{m}\left(r, r^{\prime}-r\right)+\sum_{m=q_{1}+1}^{q_{2}} \lambda_{m} D J_{m}\left(r, r^{\prime}-r\right) \geq 0
$$

Obviously, $\lambda_{0}=1$ and the construction of $\lambda_{m}^{l}$ implies that in the limit $\lambda_{m} \geq 0, m=q_{1}+1, \ldots, q_{2}$. Dividing (5.3) by $\sum_{m=0}^{q_{2}}\left|\lambda_{m}\right| \geq 1$ we can suppose that $\sum_{m=0}^{q_{2}}\left|\lambda_{m}\right|=1$. Also, if $J_{m}(r)<0$, for some $m \in\left[q_{1}+1, q_{2}\right]$, then for $l$ sufficiently large, we have $J_{m}^{l}\left(r^{l}\right)<0$ and $\lambda_{m}^{l}=0$, hence $\lambda_{m}=0$, i.e. the conditions (3.11) hold. Therefore, $r$ is also extremal.

Under the additional assumptions of Theorem 3.4 the Algorithm computes optimal controls.

Finally, we can show, see [5], that the constructed control $r_{k}^{l}$ in Step 5 of the Algorithm can be chosen to be of Gamkrelidze type and these controls can be approximated by classical controls. So, the relaxed controls can be implemented.

\section{Numerical examples}

In this section, two examples are presented. The first one without state constraints and the second one with an equality state constraint. The Algorithm applied on both problems (in the first one without penalties) with $\rho=\sigma=0.5$ and initial control $r:=\left(r_{0}+r_{1}\right) / 2$, where $r_{0}(x, t):=\delta_{0}, r_{1}(x, t):=\delta_{1}$ (Dirac measures).

Example 6.1. Let $Q:=(0,1) \times(0,1)$ and $U:=\{0,1\}$. Consider the following optimal control problem

$$
\text { minimize } J_{0}(u):=\int_{Q}\left\{0.5\left[(y-\bar{y})^{2}+|\nabla y-\nabla \bar{y}|^{2}\right]-u^{2}+u\right\} d x d t
$$

subject to

$$
\begin{gathered}
y_{t}-y_{x x}+0.5 y|y|+(1+u-\bar{u}) y=0.5 \bar{y}|\bar{y}|+\bar{y}+x(1-x)(-1+t)+2-2 t+t^{2}+\sin y-\sin \bar{y}+3(u-\bar{u}) \text { in } Q, \\
y(0, t)=y(1, t)=0, \\
y(x, 0)=x(1-x) \text { in }(0,1),
\end{gathered}
$$

and the control constraints $u \in U$, where

$$
\begin{gathered}
\bar{u}(x, t):=\left\{\begin{array}{l}
1, \text { if } 0 \leq t \leq 0.5 \\
1-2(t-0.5)(-0.4 x+0.7), \text { if } 0.5<t \leq 1
\end{array}\right. \\
\bar{y}(x, t):=x(1-x)\left(1-t+0.5 t^{2}\right) .
\end{gathered}
$$

It is easy to verify that

$$
r(x, t)\{1\}=\bar{u}(x, t), \quad r(x, t)\{0\}=1-r(x, t)\{1\}, \quad(x, t) \in Q,
$$

is the unique optimal relaxed control distributed between the points 0 and 1 with optimal state $\bar{y}$ and optimal cost 0 .

These are the results when the Algorithm was applied for 90 iterations.

$$
J_{0}^{n}\left(r_{k}^{n}\right)=3.5376 \cdot 10^{-5}, \quad d_{k}=-1.2321 \cdot 10^{-4},
$$

where $d_{k}$ was defined in Step 2 of the Algorithm. Figure 6.1 shows the last control probability function $p_{1}(x, t):=r_{k}^{n}(x, t)\{1\}$. The state y for the final iteration is shown in Figure 6.2.

Example 6.2. Consider the above problem under the equality state constraint

$$
J_{1}(u):=\int_{Q} y d x d t=0
$$

These are the results when the Algorithm was applied for 210 iterations.

$$
J_{0}^{n}\left(r_{k}^{n l}\right)=8.3807 \cdot 10^{-2}, \quad J_{1}^{n}\left(r_{k}^{n l}\right)=3.8048 \cdot 10^{-5}, \quad d_{k}=-3.8013 \cdot 10^{-3} .
$$

Here, $p_{1}(x, t):=r_{k}^{n l}(x, t)\{1\}$ is shown in Figure 6.3 and the state $y$ for the final iteration in Figure 6.4. 


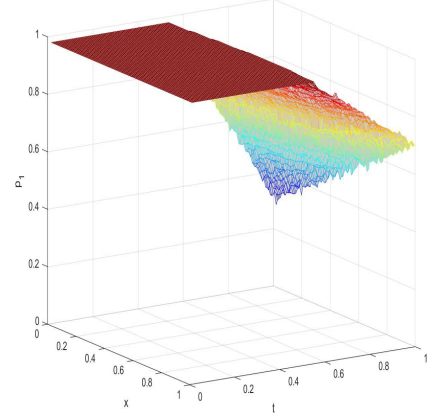

Figure 6.1: Example 6.1: Last relaxed control probability $p_{1}$

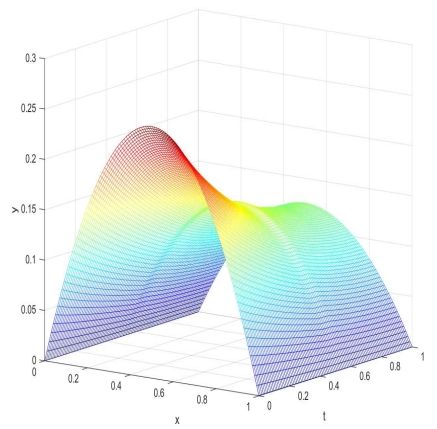

Figure 6.2: Example 6.1: State $y$ for the final iteration

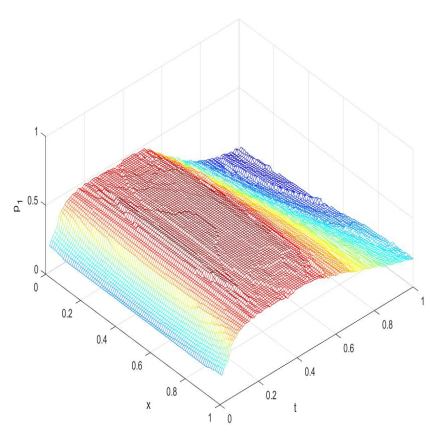

Figure 6.3: Example 6.2: Last relaxed control probability $p_{1}$

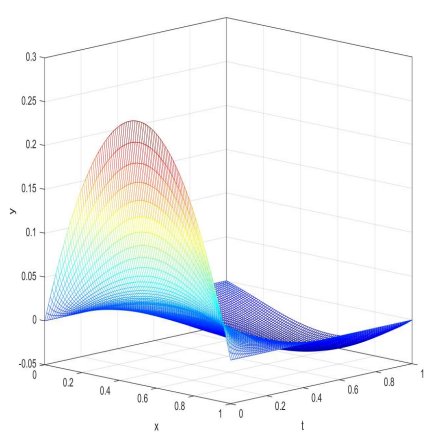

Figure 6.4: Example 6.2: State $y$ for the final iteration 


\section{Conclusion}

In the absence of any convexity assumptions, relaxed controls are an important tool to prove existence of optimal controls. Thus, the corresponding relaxed optimal control problem is introduced, which is then discretized and the behavior in the limit of sequences of optimal and admissible extremal controls was studied. Finally, a penalized conditional descent method using relaxed controls, applied to the discrete relaxed problem, is proposed. This method constructs discrete Gamkrelidze controls which, for implementation reasons, can be approximated by piecewise classical ones.

\section{References}

[1] J. Warga, Optimal Control of Differential and Functional Equations, Academic Press, New York, 1972

[2] T. Roubíček, Relaxation in Optimization Theory and Variational Calculus, Walter de Gruyter, Berlin, 1997.

[3] H. O. Fattorini, Infinite Dimensional Optimization Theory and Optimal Control, Cambridge Univ. Press, Cambridge, 1999.

[4] J. Warga, Steepest descent with relaxed controls, SIAM J. Control Optim., 15 (1977), 674-682.

[5] I. Chryssoverghi, A. Bacopoulos, B. Kokkinis, J. Coletsos, Mixed Frank-Wolfe penalty method with applications to nonconvex optimal control problems, J. Optimiz. Theory App., 94 (1997) 311-334.

[6] I. Chryssoverghi, A. Bacopoulos, Approximation of relaxed nonlinear parabolic optimal control problems, J. Optimiz. Theory App., 77 (1993) $31-50$.

[7] T. Roubíček, A convergent computational method for constrained optimal relaxed control problems, J. Optimiz. Theory App., 69 (1991) 589-603.

[8] V. Azhmyakov, W. Schmidt, Approximations of relaxed optimal control problems, J. Optimiz. Theory App., 130 (2006) 61-77.

[9] N. Arada, J. P. Raymond, State-constrained relaxed problems for semilinear elliptic equations, J. Math. Anal. Appl., 223 (1998) $248-271$.

[10] N. Arada, J. P. Raymond, Approximation of optimal control problems with state constraints, Numer. Funct. Anal. and Optimiz., 21 (2000) 601-621.

[11] E. Casas, The relaxation theory applied to optimal control problems of semilinear elliptic equations, J. Dolezal, J. Fidler (editors), System Modelling and Optimization, Chapman \& Hall, London, 1996, pp. 187-194.

[12] I. Chryssoverghi, J. Coletsos, B. Kokkinis, Discrete relaxed method for semilinear parabolic optimal control problems, Control Cybernet., 28 (1999)

[13] S. Luan, Nonexistence and existence of an optimal control problem governed by a class of semilinear elliptic equations, J. Optimiz. Theory App., $\mathbf{1 5 8}$ (2013) $1-10$

[14] I. Chryssoverghi, J. Coletsos, B. Kokkinis, Classical and relaxed optimization methods for nonlinear parabolic optimal control problems I. Lirkov, S. Margenov and J. Waśniewski (editors), Large-scale scientific computing, Springer-Verlag, Berlin, 2010, pp. $247-255$.

[15] J. L. Lions, Quelques Méthodes de Résolution des Probèmes aux Limites Non Linéaires, Dunod Gauthier-Villars, Paris, 1969.

[16] R. Temam, Navier-Stokes Equations, North-Holland, New York, 1977.

[17] S. Treanta, On signomial constrained optimal control problems, Commun. Adv. Math. Sci., 2 (2019), 55-59. 